\title{
Direct Synthesis of 2D-Hexagonal Mesoporous Iron Silicate and its Catalytic Activity for Selective Friedel-Crafts Alkylation
}

\author{
Swapan K. Das, Manas K. Bhunia and Asim Bhaumik*
}

Department of Materials Science, Indian Association for the Cultivation of Science, Jadavpur, Kolkata - 700032, India

\begin{abstract}
D-hexagonal mesoporous iron silicate (HMFeS) has been synthesized hydrothermally in the presence of a mixture of an amphiphilic triblock copolymer, pluronic F127 and 1,2,4-trivinylcyclohexane (TVCH) as swelling agent under acidic aqueous conditions. The direct incorporation of iron(III) into 2D-hexagonal silicate framework can be monitored in a optimized molar ratio of water and hydrochloric acid. The mesophase of the materials was investigated by using small-angle powder X-ray diffractions (PXRD), transmission electron microscopy (TEM) image analysis and nitrogen adsorption/desorption studies. TEM image and PXRD revealed that the material had 2D-hexagonal mesoporous architecture. The morphology of the material was investigated by using scanning electron microscope (SEM) and framework bonding by utilizing FT IR spectroscopy. The atomic absorption spectrophotometer (AAS) was used to estimate the incorporated iron sites within the silicate framework. BET surface area $\left(780 \mathrm{~m}^{2} \mathrm{~g}^{-1}\right)$ and peak pore size of HMFeS $(10.07 \mathrm{~nm})$ is much higher than the pure silica SBA-15 $\left(611 \mathrm{~m}^{2} \mathrm{~g}^{-1}\right.$ and peak pore size of $\left.9.09 \mathrm{~nm}\right)$. This mesoporous material (HMFeS) acts as a very good catalyst in the Friedel Craft benzylation and benzoylation reactions of arenes under optimized reaction condition using benzyl chloride and benzoyl chloride as the bezylating and benzoylating agents, respectively.
\end{abstract}

Keywords: 2D-hexagonal, Mesoporous silica, Iron, Friedel-Craft benzylation and benzoylation.

\section{INTRODUCTION}

Mesoporous materials have engrossed mammoth in-depth look to the scientific community due to their outstanding structural and textural features like high surface area, large pore volume, ordered pore networks with uniform and welldefined pore architecture since their invention [1,2]. These features have made mesoporous materials as potential candidates for versatile applications including gas storage $[3,4]$, adsorption/ separation [5,6], catalysis [7-11], sensors $[12,13]$, solar cells [14-16] optoelectronics [17-19] and many other frontier areas of science. As a prototypical mesoporous material, purely siliceous SBA-15 material possesses larger pore diameter and relatively thicker pore walls, offers high thermal stability and hydrothermal stability than those for MCM-41 [20,21]. All these properties of SBA-15 material make it attractive and robust material for catalytic applications [20-24]. Nevertheless, the most noteworthy drawbacks of the SBA-15 material are that there are few catalytically active sites on its amorphous $\mathrm{SiO}_{2}$ wall [25] in comparison to zeolites, which usually possess a large number of isolated active sites [26, 27]. Thus, it is highly desirable to incorporate metal ion other than $\mathrm{Si}^{4+}$ to the surface of the SBA-15 porous framework for its possible usage as catalyst [28].

Several synthetic strategies are available for the incorporation of transition metals into mesoporous silicate

*Address correspondence to this author at the Department of Materials Science, Indian Association for the Cultivation of Science, Jadavpur, Kolkata - 700 032, India; Tel: +913324734971, Ext. 207; Fax: +91 332473 2805; E-mail: msab@iacs.res.in frameworks [28-30]. Recently, extensive efforts have been given to engineer different heteroelements like bivalent $(\mathrm{Cu}$, $\mathrm{Co}, \mathrm{Ni}, \mathrm{Zn}$, etc.), trivalent (Al, $\mathrm{Fe}, \mathrm{Ga}$, etc.) or tetravalent $(\mathrm{Ti}, \mathrm{Zr}, \mathrm{Sn}$, etc.) incorporated mesoporous silicate frameworks [28-33] having large pore that essentially used as catalyst for different interesting reactions involving large molecules, as large molecules are not able to enter into the channels of microporous zeolites. The direct hydrothermal method is one of the most accepted methods of incorporating metals into mesoporous silica, which consists of the direct addition of metal ion precursors to the synthesis gel prior to the hydrothermal treatment [30, 34]. Other conventional methods like wet impregnation and grafting $[24,35,36]$ have also been used though these methods have faced several problems including the loss of the ordered mesoporous structure, low metal loadings, or formation of bulk species outside the silica host [37]. This direct synthesis method often requires specialized synthesis conditions depending on the respective structures of the materials, and the incorporation of iron into the silica matrix usually causes a decrease in the structural ordering [37]. The purely siliceous phase of SBA-15 like material is usually synthesized in strong acidic media (2 $\mathrm{M} \mathrm{HCl}$ solution) [20] and most of the iron sources dissolve in strong acids, thus the incorporation iron into silicate framework by direct synthesis seems unlikely.

The liquid-phase benzylation of benzene and other aromatic compounds using benzyl chloride is important for the production of diphenylmethane and substituted diphenylmethanes, which are being industrially important as intermediates for pharmaceuticals and other fine chemicals [38-41]. Conventional strong Lewis acid catalysts, such as 
$\mathrm{ZnCl}_{2}, \mathrm{AlCl}_{3}, \mathrm{NiCl}_{2}, \mathrm{CoCl}_{2}, \mathrm{SnCl}_{4}, \mathrm{FeCl}_{3}, \mathrm{BF}_{3}$, and BrØnsted acid like $\mathrm{H}_{2} \mathrm{SO}_{4}$ are utilized in homogeneous conditions for such type of Friedel-Crafts reactions. However, the use of those catalysts meets a lot of disadvantages due to their toxicity, handling hazards, their requirement in stoichiometric amounts, difficulty in separation and recovery of the catalyst. Moreover, there are also limitations of product selectivity due to formation of complex mixture, large amounts of salt as by-products and hydrogen halides often leading to production of other side products. Thus, there has been much interest in replacing these homogeneous catalysts with heterogeneous solid acid catalysts possessing high activity for Friedel- Crafts type reactions, and which can be separated easily from the reaction mixture and reused. Choudhary et al. found that $\mathrm{AlCl}_{3}$ supported on Si-MCM-41 had poor catalytic activity for the benzylation of benzene rather than the supported $\mathrm{FeCl}_{3}, \mathrm{GaCl}_{3}$, and $\mathrm{InCl}_{3}$ [42-44]. On the contrary, $\mathrm{Hu}$ et al. have explored that $\mathrm{AlCl}_{3} / \mathrm{MCM}-41$ showed higher activity compared to other metal chloride supported mesoporous catalysts, such as, $\mathrm{NiCl}_{2} / \mathrm{MCM}-41$, $\mathrm{FeCl}_{3} / \mathrm{MCM}-41, \mathrm{ZnCl}_{2} / \mathrm{MCM}-41$ and $\mathrm{CuCl}_{2} / \mathrm{MCM}-41$ [45]. Nevertheless, the immobilized metal chloride blocks the pores, either partially or fully, thereby reducing the specific surface area, pore volume, and pore diameter, and can also play a negative role in the catalysis by leaching, catalyst poisoning, or obstruction of the active sites.

In the present work, we have demonstrated that the hydrothermal synthesis of iron incorporated silicate materials by direct addition of iron source to the synthesis gel prior to the hydrothermal treatment in presence of a mixture of pluronic F127 and 1,2,4-trivinylcyclohexane as swelling agent under acidic aqueous condition. The method described in this work is a simple and new approach for the synthesis of Fe-containing mesoporous silica materials. The mesophase of the material was investigated by using smallangle PXRD, TEM image analysis and nitrogen sorption studies. The HMFeS material suppose to have moderate acidity and are potential catalysts for many catalytic reactions that do not require strong acid sites, especially for Friedel-Crafts alkylation and acylation reactions involving large molecules [46]. This HMFeS material acts as a superior catalyst in the Friedel Craft benzylation as well as benzoylation reactions of arenes using benzyl chloride and benzoyl chloride as bezylating and benzoylating agents, respectively.

\section{EXPERIMENTAL SECTION}

\section{Chemicals}

Triblock copolymer poly(ethylene glycol)-block-poly(propylene glycol)-block-poly(ethylene glycol) (Pluronic F127, $\left.M_{\mathrm{av}}=12600, \quad \mathrm{EO}_{106} \mathrm{PO}_{70} \mathrm{EO}_{106}\right)$ and 1,2,4trivinylcyclohexane (TVCH) was used as the structuredirecting agent (SDA) and co-surfactant, respectively and were purchased from Sigma-Aldrich. Tetraethyl orthosilicate (TEOS, silica source) and diphenylmethane were purchased from Sigma-Aldrich. Iron(III) nitrate nonahydrate $\left[\mathrm{Fe}\left(\mathrm{NO}_{3}\right)_{3}\right.$, $\left.9 \mathrm{H}_{2} \mathrm{O}\right]$, hydrochloric acid $(\mathrm{HCl})$, potassium chloride $(\mathrm{KCl})$ and benzene (caution: poisonous and carcinogenic) and different arenes, benzyl chloride and benzoyl chloride were obtained from Merck. All chemicals were used without further purification.

\section{Synthesis}

Iron incorporated large mesoporous iron silicate material has been synthesized through the following procedure. In the typical synthesis, $25 \mathrm{ml}$ of distilled water was acidified with $0.340 \mathrm{~g}(35 \mathrm{wt} \%)$ hydrochloric acid. Then, $1.0 \mathrm{~g}$ of pluronic F127 was added to this acidic aqueous medium and allowed to stir until dissolution. After complete dissolution, $2.50 \mathrm{~g}$ of potassium chloride $(\mathrm{KCl})$ was added to balance the ionic strength of the solution. Then, $1.3 \mathrm{~g}$ of 1,2,4trivinylcyclohexane was added slowly as swelling agent [47] and then allowed to stir for overnight. Then, it was aged at room temperature for 1 day and $2.33 \mathrm{~g}$ tetraethyl orthosilicate (TEOS) was added slowly to the micellar solution and allowed to stir for $4 \mathrm{~h}$. Then, $0.9061 \mathrm{~g}$ iron(III) nitrate nonahydrate taken in $5 \mathrm{ml}$ water was added to that solution under vigorous stirring and total mixture was allowed to stir for another $4 \mathrm{~h}$. After that the mixture was covered and aged for 7 days at ambient temperature. The molar composition of the synthesis gel mixture was 1: 0.0260.098: 2.99: 0.714: 0.006: 1.04: 148.2 TEOS: $\mathrm{Fe}_{2} \mathrm{O}_{3}: \mathrm{KCl}$ : TVC: F127: $\mathrm{HCl}: \mathrm{H}_{2} \mathrm{O}$. Then, it was transferred into an autoclave and allowed to hydrothermal treatment at $373 \mathrm{~K}$ for $24 \mathrm{~h}$. After cool down slowly to the room temperature, the resultant solid was filtered, washed with distilled water, dried at $323 \mathrm{~K}$. Calcination was carried out by slowly increasing temperature to $873 \mathrm{~K}\left(1 \mathrm{~K} \mathrm{~min}^{-1}\right.$ ramping rate) and by heating at $873 \mathrm{~K}$ for $5 \mathrm{~h}$ in presence of air to obtain template-free large mesoporous iron silicate catalyst. Spectroscopic analysis suggests that iron incorporation is only possible at optimized reaction condition, maintaining the $\mathrm{pH}$ of the synthesis gel at ca. 2.0. We have already prepared pure silicate material by utilizing pluoronic F127 (as SDA) and 1,2,4-trivinylcyclohexane (TVCH, as swelling agent) in a strongly acidic aqueous medium [33].

\section{Characterization Techniques}

Powder X-ray diffraction patterns (XRD) of the samples were recorded on a Bruker AXS D-8 Advance diffractometer operated at $40 \mathrm{kV}$ voltage and $40 \mathrm{~mA}$ current and calibrated with a standard silicon sample, using Ni-filtered $\mathrm{Cu} \mathrm{K \alpha}(\lambda=$ $0.15406 \mathrm{~nm}$ ) radiation. Transmission electron microscopic images were recorded on a JEOL 2010 TEM operated at 200 $\mathrm{kV}$. A Jeol JEM 6700 field emission scanning electron microscope (FE-SEM) was used for the determination of morphology of the particles. Nitrogen adsorption/desorption isotherms of the samples were recorded on a Quantachrome Autosorb 1C, at $77 \mathrm{~K}$. Prior to the gas adsorption measurements, the samples were degassed at $473 \mathrm{~K}$ for $6 \mathrm{~h}$ under high vacuum. Atomic absorption spectroscopic (AAS) analyses were carried out by using a Shimadzu AA-6300 spectrometer fitted with a double beam monochromator. Fourier transform infrared (FT IR) spectra of these samples were recorded on $\mathrm{KBr}$ pellets by using Perkin-Elmer Spectrum 100 spectrophotometer.

\section{Catalytic Conditions}

Catalytic Friedel-Crafts benzylation and benzoylation of different arenes were carried out by utilizing benzyl chloride and benzoyl chloride as the benzylating, benzoylating agents, respectively in a two neck round bottom flask fitted with a water condenser and placed in an oil bath at $348 \mathrm{~K}$ under magnetic stirring. The temperature of the oil bath was 
kept constant. During these experiment, arenes and benzyl chloride or benzoyl chloride have been taken in stoichiometric ratio of $15: 1$ and $10: 1$ and $1 \mathrm{wt} \%$ of catalysts has been taken with respect to benzyl chloride or benzoyl chloride. In a typical run, arenes and benzyl chloride or benzoyl chloride have been taken in a mentioned ratio, and desired amount of catalyst was added immediately into this. In the cases at the selected time intervals the products were collected from the reaction mixtures and analyzed by capillary gas chromatography (Agilent 4890D, FID) fitted with a capillary column; $0.5 \mathrm{ml} n$-heptane was introduced to each of the reaction mixtures as internal standard before analysis. Products were identified from known standards and mass spectrometric analysis by using a Micromass Q-tofMicro Quadruple mass spectrophotometer. Since large excess of arenes have been taken in these reactions, so percentage of conversion was estimated by taking respective benzyl chloride or benzoyl chloride as standard.

\section{RESULTS AND DISCUSSION}

Typically, iron is incorporated into the framework of a silicate material via a so-called "direct synthesis" procedure in which an iron precursor is added into the gel prior to hydrothermal synthesis. Herein, we present a synthesis strategy of large mesoporous iron silicate where iron incorporation is largely influenced by molar ratio of hydrochloric acid to water as well as amount of iron input in the synthesis gel. Positively charged iron hydroxo complexes $\left[\mathrm{Fe}\left(\mathrm{H}_{2} \mathrm{O}\right)_{5} \mathrm{OH}\right]^{2+}$ can effectively interact with negatively charged silicate above isoelectric point of silica $(\mathrm{pH} \sim 2)$ leading to the incorporation of iron into the hexagonal silicate framework [30]. It has been observed that the incorporation of iron in the solid phase is some extent less than the synthesis gel composition due to high solubility of the iron precursors in the acidic aqueous medium.

\section{Mesophase Analysis}

The Small angle powder X-ray diffraction (XRD) patterns of the calcined mesoporous pure silicate and iron silicate materials are shown in Fig. (1). The material shows three well-resolved diffraction peaks in the region of $2 \theta=$ $0.66-1.7^{\circ}$, which can be indexed in turn to the 100,110 and 200 reflections corresponding to a two-dimensional hexagonal mesostructure with space group $\mathrm{p} 6 \mathrm{~mm}$ and high

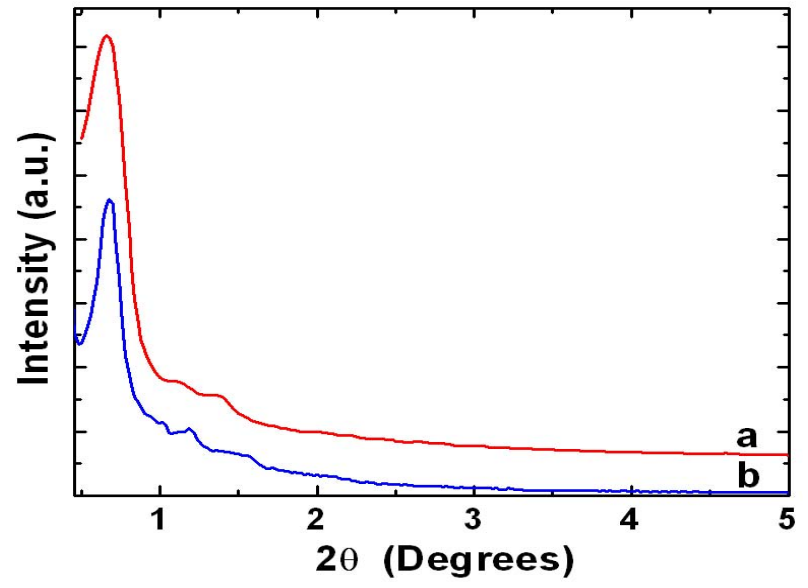

Fig. (1). Small-angle powder XRD patterns of mesoporous SBA-15 (a) and HMFeS materials (b). structural periodicity [20]. The higher order diffraction peak was too weak due to incorporation of heteroatoms into the framework [48]. The periodically ordered two dimensional (2-D) hexagonal mesostructure with $d$ value $14.2 \mathrm{~nm}$ is further confirmed by TEM characterization as shown in Fig. (2) [30]. The high angle XRD pattern (not shown here) shows no higher order diffraction peaks or no characteristic peaks of $\mathrm{Fe}_{2} \mathrm{O}_{3}$, indicating the amorphous nature of the pore walls [48]. Thus, it is concluded that iron might be in the framework or highly disperse throughout the silicate surface [34]. The slight reduction of the 100 reflections in the iron loaded Fe-SBA-15 sample is more likely due to a dilution of silica with incorporation of iron as a consequence of higher adsorption factor for X-rays than silicon [49].

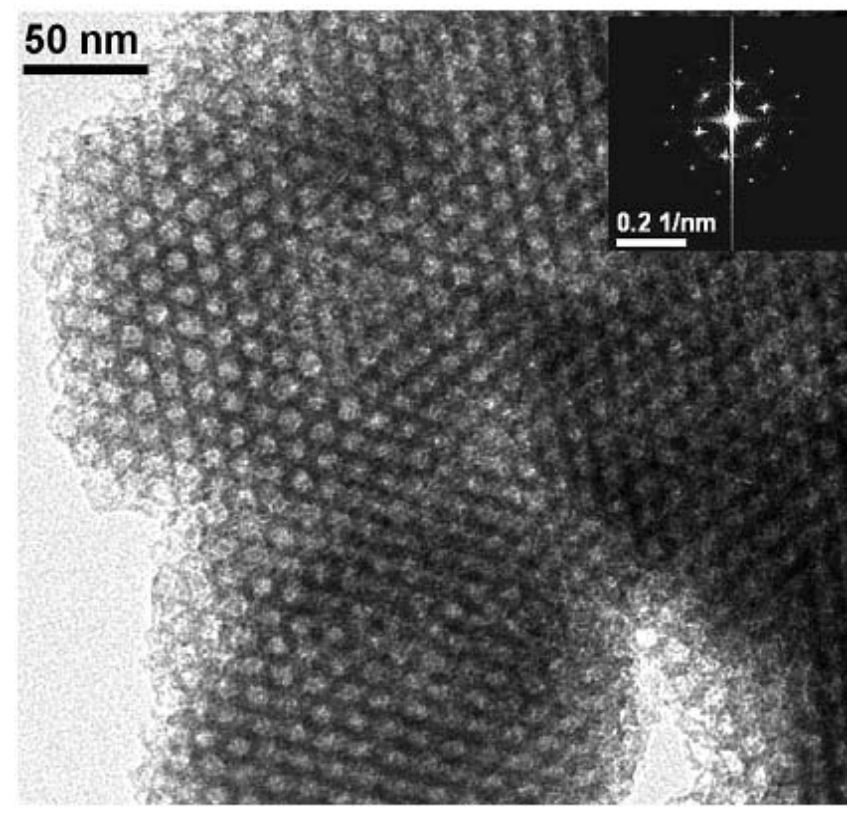

Fig. (2). TEM image of HMFeS material. Pores are seen through the direction perpendicular to pore axis of the material.

\section{Nanostructure and Porosity}

Representative TEM image of the HMFeS sample is shown in Fig. (2). In the image low electron density spots (pores) are seen throughout the specimen. TEM image and corresponding FFT diffractogram (inset of Fig. 2) are recorded along (100) direction indicating uniform long range ordering, where pores are hexagonally arranged [34]. In the case of pure silicate material, pores are uniform in size of $c a$. $9.09 \mathrm{~nm}$ and the wall-thickness varies from 5.0 to $5.8 \mathrm{~nm}$ [33]. Whereas, in case of iron silicate pores are also uniform in size of about $10.07 \mathrm{~nm}$ and wall thickness vary from 4.0 to $4.5 \mathrm{~nm}$. Thus, incorporation of iron into the silicate framework makes the pore walls slimmer than the pure silica. Consequently, the material becomes lighter. Average pore diameters measured from this micrograph are well consistent with the BJH pore size distribution derived from $\mathrm{N}_{2}$ adsorption isotherms as shown in Fig. (4). Field emission scanning electron microscope (FE-SEM) image of HMFeS (shown in Fig. 3) reveals that sample is composed of small particles. These small particles are agglomerated together to form a large size of spheres with diameters ranging from 7 to $12 \mu \mathrm{m}$ 


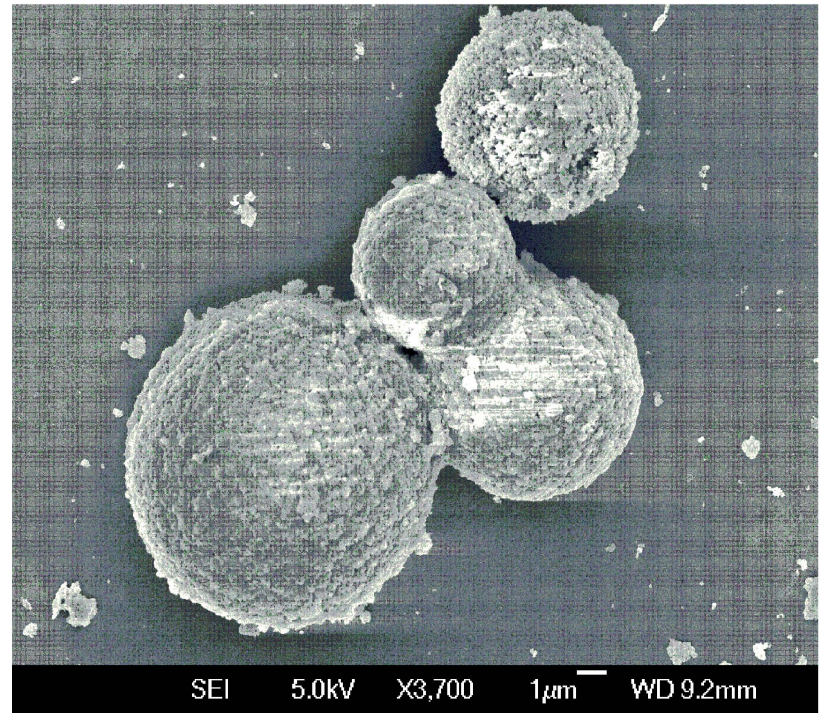

Fig. (3). FE SEM images of mesoporous HMFeS material.

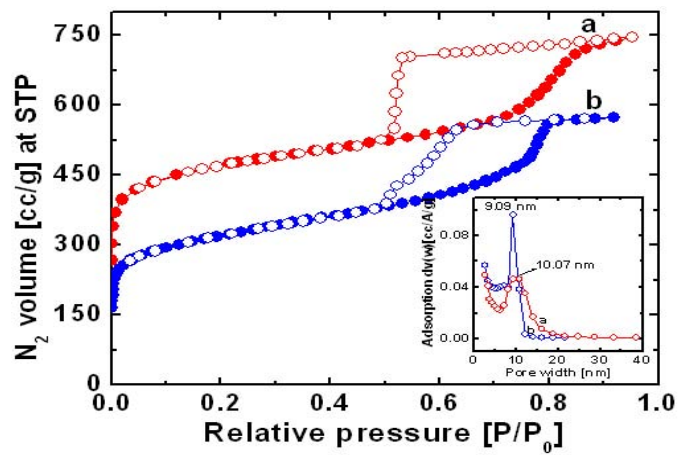

Fig. (4). $\mathrm{N}_{2}$ adsorption/desorption isotherms of mesoporous SBA15 (a) and HMFeS materials (b) measured at $77 \mathrm{~K}$. Y axis of plot a and $\mathrm{b}$ have been enhanced by 250 and 150 , respectively for clarity. Adsorption points are marked by filled circles and desorption points by empty circles. Respective pore size distributions using $\mathrm{BJH}$ method are shown in inset of Fig. (4).

Nitrogen-sorption studies carried out on the calcined mesoporous silica and $\mathrm{HMFeS}$ materials at $77 \mathrm{~K}$, shows a type-IV adsorption-desorption isotherm with a very large $\mathrm{H}_{2}$ type hysteresis loop in the $P / P_{0}$ range from 0.60 to 0.82 as shown in Fig. (4). These isotherms suggesting large uniform mesopores with a cage-like pore structure connected by windows with a small size and confirming that the samples are mesoporous [30,34,47]. This is the characteristic of capillary condensation observed for uniform mesopores. The $P / P_{0}$ position of the inflection points is clearly related to a diameter in the mesopore range, and the sharpness of these steps indicates the distributions of the mesopores are uniform in size [50]. Brunauer-Emmett-Teller (BET) surface area, average pore diameter, and pore volume, of these samples estimated from their respective adsorption-desorption isotherms are given in Table 1. The BET surface area of the calcined mesoporous silica and HMFeS were $611,780 \mathrm{~m}^{2} \mathrm{~g}^{-1}$, respectively [33]. Here, BET surface area of HMFeS (780 $\left.\mathrm{m}^{2} \mathrm{~g}^{-1}\right)$ is sufficiently higher than pure silica $\left(611 \mathrm{~m}^{2} \mathrm{~g}^{-1}\right)$. Because, iron incorporation into silicate framework makes the pore walls slimmer than pure silica, which is reflected in their surface areas and also confirmed by their respective TEM images (Fig. 2) [33]. Pore size distribution of the pure silica is $9.09 \mathrm{~nm}$, where as $10.07 \mathrm{~nm}$ for HMFeS estimated by employing the BJH method, which agree quite well with the pore widths obtained from the TEM image (Fig. 2) and also XRD analysis (Fig. 1).

\section{Spectroscopic Analysis}

FT IR spectra of the calcined SBA-15 and LHMFeS samples are shown in Fig. (5). The absence of the bands at $~$ 2854 and $\sim 2925 \mathrm{~cm}^{-1}$ for the calcined samples, which are ascribed to symmetric and asymmetric vibrations of the $\mathrm{C}-\mathrm{H}$ groups, indicates the complete removal of F127 molecules from both the as-synthesized materials after calcinations. The broad band $\sim 3500$ and $1650 \mathrm{~cm}^{-1}$ are attributed to the asymmetric $\mathrm{OH}$ stretching and bending of water molecule, respectively. The slightly red shifting of the band from 1087 $\mathrm{cm}^{-1}$ to $1078 \mathrm{~cm}^{-1}$ observed due to the incorporation of iron within the silica network which generally corresponds to the stretching vibration of the tetrahedral $\mathrm{SiO}_{4}$ units [51]. This shifting indicated that heteroatom (iron) was incorporated into the silicate framework and $\mathrm{Fe}-\mathrm{O}-\mathrm{Si}$ bonds were formed. It is well known fact that the bond length of Fe-O was larger than that of Si-O bonds, which led to a decrease in the force constant $(\mathrm{k})$ of the band. Furthermore, the atomic weight of iron is higher than that of $\mathrm{Si}$, so reduced mass (1) was increased, hence these factors collectively shifted the vibration frequencies to the lower range and which was observed in this material. In contrast, this phenomenon did not exist for the bands at $c a .460 \mathrm{~cm}^{-1}$, which corresponded to the bending vibration of surface $\mathrm{Si}-\mathrm{O}^{-}$bond present in the material.

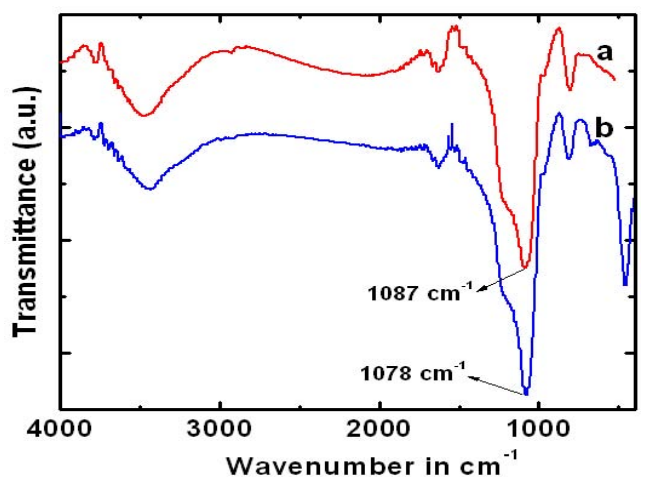

Fig. (5). FT IR spectra of mesoporous SBA-15 (a) and HMFeS (b) materials.

Table 1. Physico-Chemical Properties of SBA-15 and HMFeS Materials

\begin{tabular}{|c|c|c|c|c|}
\hline Entry & Sample Name & Surface Area $\left(\mathbf{m}^{\mathbf{2}} \mathbf{g}^{-1}\right)$ & Pore Width (nm) & Pore Volume (ccg $\left.{ }^{-1}\right)$ \\
\hline \hline 1 & SBA-15 & 611 & 9.09 & 0.64 \\
\hline 2 & HMFeS & 780 & 10.07 & 0.50 \\
\hline
\end{tabular}




\section{Catalysis}

The catalytic performance of HMFeS was investigated in the Friedel Crafts benzoylation reactions of different arenes with benzoyl chloride as benzoylating agent as shown in Table 2. This reaction was carried out at optimized temperature of $348 \mathrm{~K}$ by taking arenes to benzoyl chloride molar ratio of 10:1. The catalyst is so efficient that only 1 wt $\%$ catalyst (HMFeS) with respect to benzoyl chloride can efficiently convert almost $100 \%$ product within few hours as shown in the Table 2 . The calculated conversion of benzoyl chloride to the respective benzoylated product is based on the $100 \%$ selectivity. This HMFeS material showed very good catalytic activity in the Friedel-Craft benzoylation reaction due to very fine dispersion of Fe-sites at the mesopore surface of the framework. During benzoylation reaction benzoyl chloride generates benzoylium ion intermediate in presence of iron within HMFeS material as shown in Scheme 1A. For the sample with molar ratio of $\mathrm{n}_{\mathrm{Si}} / \mathrm{n}_{\mathrm{Fe}}$ of 24.0 give efficient transformation of arenes to benzoylated compounds because in this ratio plenty of active (surface disperse Fe-sites) sites are available, which provides catalytic sites for the Friedel-Craft alkylation. When the iron content in the materials exceeded the limitation, the deposited $\mathrm{Fe}_{2} \mathrm{O}_{3}$ covered some of the active iron species and

Table 2. Benzoylation of Different Arenes with Benzoyl Chloride Catalyzed by HMFeS Material ${ }^{\mathrm{a}}$

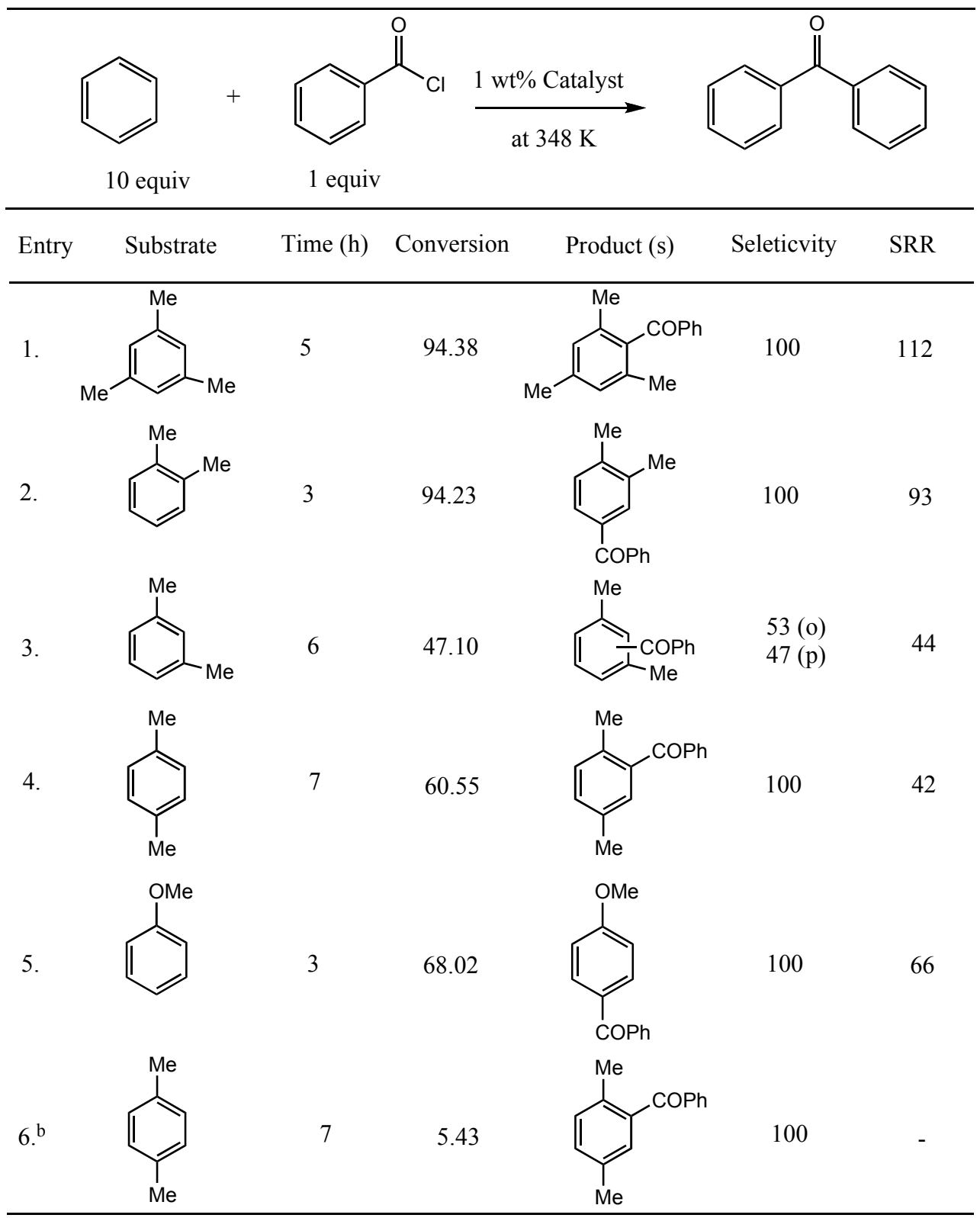

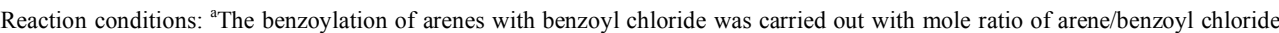
of 10:1 and 5:1. 5 mmole benzoyl chloride and 50 mmole s (10:1 ratio) arene and 25 mmole (5:1 ratio) arenes. Here, arenes have been taken as large excess because those acts as reactant as well as solvent. Temperature of the reaction was maintained at $348 \mathrm{~K}$. Catalyst was taken $1 \%(\mathrm{w} / \mathrm{w})$ based on benzoyl chloride. SRR (specific reaction rate) $=$ moles of substrate converted $/ \mathrm{moles}$ of Fe present in the catalyst. Assuming all Fe-sites are located at the catalyst surface.

benzoylation reaction was carried out without catalyst. In every case product selectivity was $100 \%$ and only in case of m-Xylene two products were obtained. 
<smiles>O=C(Cl)C1=C[C+]=CC(=Cc2cccc(C(=O)Cl)c2)C1</smiles>

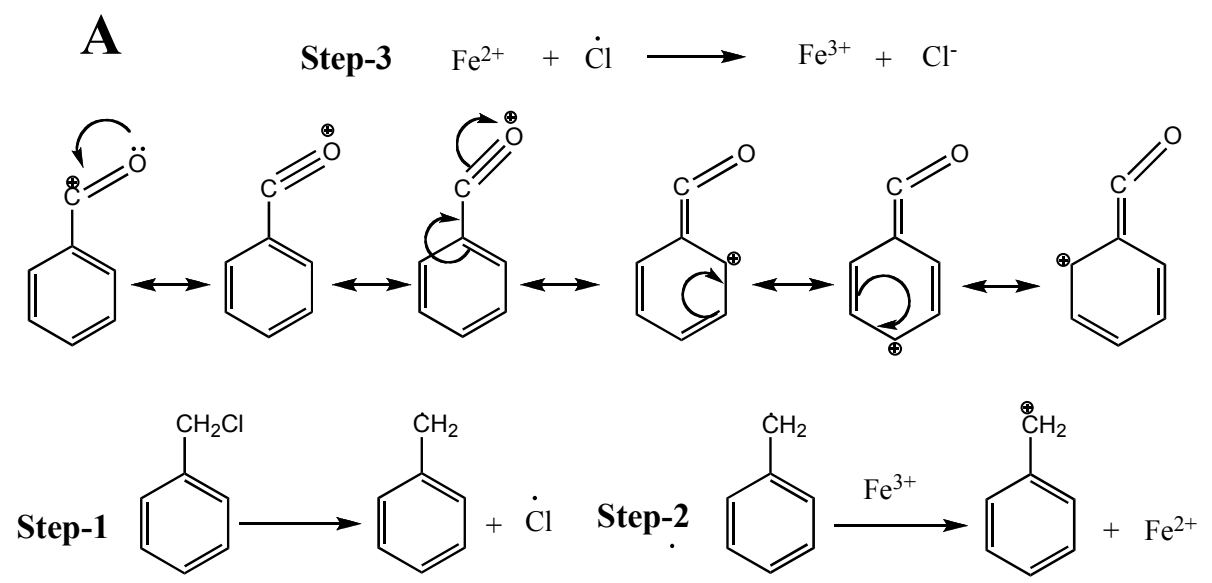

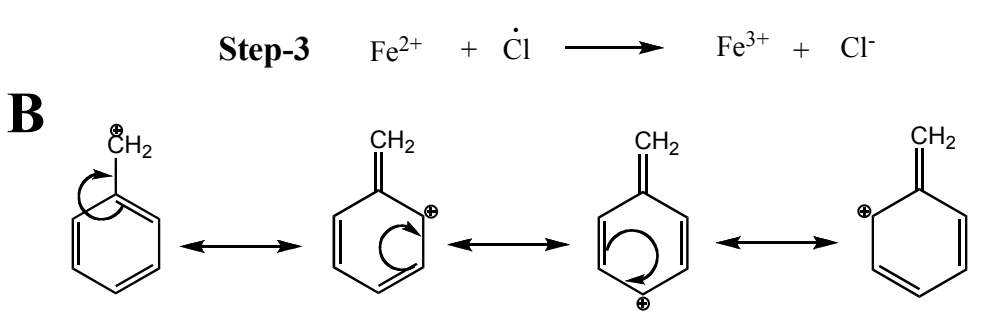

Scheme 1. Schematic representation of redox mechanism for the activation of (A) benzoyl chloride during benzoylation reaction and (B) benzyl chloride during benzylation reaction in presence of HMFeS catalyst. 2D-hexagonal mesoporous iron silicate (HMFeS) has been synthesized hydrothermally in the presence of a mixture of an amphiphilic triblock copolymer, pluronic F127 and 1,2,4-trivinylcyclohexane (TVCH) under acidic aqueous condition and the material showed superior catalytic activity in the Friedel-Craft benzylation and benzoylation reactions.

hampered the active sites, which led to the decrease of catalytic activity. When we take mesitylene and o-xylene as substrates, almost $100 \%$ conversion occurs, forming monobenzoylated product. In case of mesitylene and oxylene, "+I" effect of methyl group activates the benzene ring to benzoylation reaction and all positions are identical with respect to methyl group, resulting $100 \%$ selective product. But in case of anisole, " $+\mathrm{R}$ " effect of methoxy group activates the para position of the ring leading to $100 \%$ selective product.

Moreover, the Fridel Crafts benzylation of different arenes with benzyl chloride as benzylating agent were carried out at optimized temperature of $348 \mathrm{~K}$ by taking molar ratios of arenes to benzyl chloride $15: 1$ and 10:1 in presence of HMFeS as shown in Tables $\mathbf{3}$ and $\mathbf{4}$, respectively. Only $1.0 \mathrm{wt} \%$ of the catalyst with respect to benzyl chloride can efficiently convert almost $100 \%$ monobenzylated product within few hours. During benzylation reaction benzyl chloride generates benzylium ion intermediate which reacts with arenes to give corresponding monobenzylated compound in the attendance of HMFeS as shown in Scheme 1B. The specific reaction rate in all the reactions is quite high, which indicates that the catalysts are very much efficient in this reaction. Here, high surface area as well as large pore size provides the plenty of accessible Fe-dispersed sites and increases this type of reaction. The results indicate that the benzylation activity of the Fe-SBA-15 is increased in the presence of electrondonating and / or mesomeric groups such as methyl, hydroxyl, etc in the arenes, which makes the benzene moiety very much electron rich and consequently activates the ring towards benzylation. Furthermore, we use excess arene as it acts as solvent and to avoid competing di- and polybenzylation [52].

The stability as well as heterogeneous nature of mesoporous iron silicate was investigated by performing a hot filtration test utilizing the HMFeS catalyst under the optimized reaction conditions. In this case, the reaction was stopped after certain time and we estimated conversion level has reached to $40 \%$ at this stage. This is followed by quick removal of the catalyst through filtration. The filtrate was immediately reused in the same reaction in under identical reaction conditions. We have not observed any further increase of substrate conversion. Furthermore, the filtered catalyst was reused for the next cycle under same reaction conditions and no loss of its catalytic activity was observed. Thus, it is clear that in the recycles of the catalysts, the catalysts retained their activity and this is the directly informed the retention of catalytic activity in the recycling step. Moreover, when the reaction was carried out in absence of catalyst, almost no conversion was found. These results confirmed that during the reaction almost no catalyst 
Table 3. Benzylation of Different Arenes with Benzyl Chloride Catalyzed by Mesoporous Iron Silicate Material ${ }^{\mathrm{a}}$

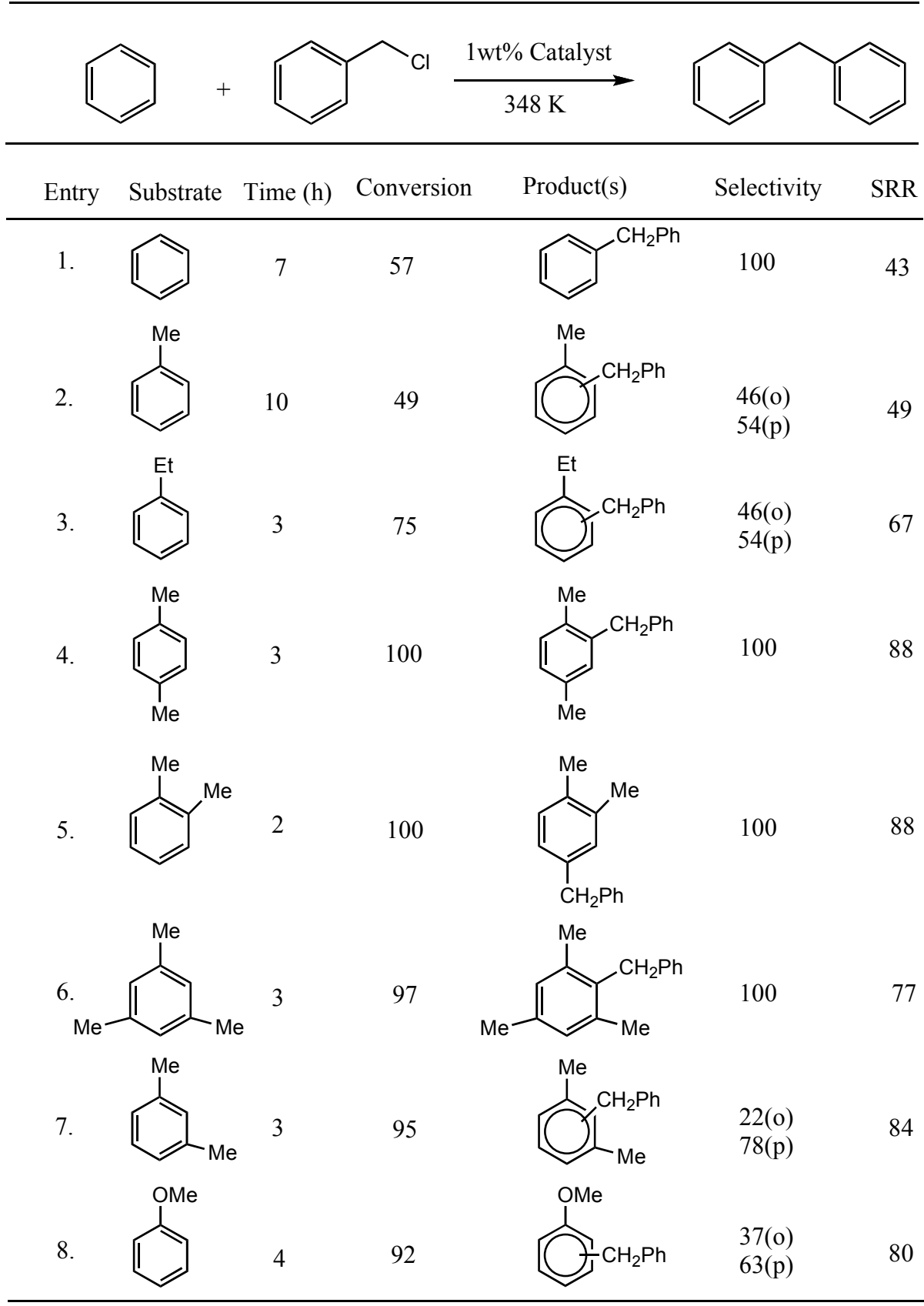

${ }^{a}$ Reaction conditions: The benzylation of arenes with benzyl chloride was carried out with mole ratio of arene/benzoyl chloride of $15: 1$.

leaching occurs and the reaction is a purely heterogeneous in nature. The activity of a regenerated catalyst was inspected by filtration of the catalyst from a standard reaction and then washing several times with methanol and anhydrous acetone followed by drying in an oven at $373 \mathrm{~K}$ for overnight. The catalyst was subsequently activated at $673 \mathrm{~K}$ for $4 \mathrm{~h}$ under air flow and utilized for the above reaction in the same reaction condition. In both type of reactions arenes have been taken as large excess, it acts as reactant as well as solvent. Hence, here the conversion was estimated with respect to benzyl chloride and benzoyl chloride reported earlier [30]. The above results suggested that our large mesoporous 2D-hexagonal iron silicate material is a superior catalyst for the production of benzoylated and benzylated derivatives of different arenes under mild liquid phase reaction conditions.

\section{CONCLUSION}

In conclusion, we have presented the new synthetic strategy for the preparation of mesoporous HMFeS through hydrothermal synthesis method using a non-ionic amphiphilic tri-block copolymer surfactant, pluronic F127 under acidic $\mathrm{pH}$ conditions. Here, combination of triblock copolymer, pluronic F127, and TVCH leads to generate such large mesoporous architecture. TEM and $\mathrm{N}_{2}$ adsorption/desorption studies revealed the mesoporous nature in these materials. We have monitored the optimized 
Table 4. Benzylation of Different Arenes with Benzyl Chloride Catalyzed by Mesoporous Iron Silicate Material

Entry Substrate Time (h) Conversion

${ }^{a}$ Reaction conditions: The benzylation of arenes with benzyl chloride was carried out with mole ratio of arene/benzyl chloride 10:1 (Table 4). 5 mmole of benzyl chloride and 50 mmole of arenes have been taken in each reaction. Here arenes have been taken as large excess compare to benzoyl chloride because arenes acts as solvent as well as reactant. Temperature of the reaction was maintained at $348 \mathrm{~K}$. Catalyst was taken $1 \%(\mathrm{w} / \mathrm{w})$ based on benzyl chloride.

${ }^{b}$ Benzylation reaction was carried out without catalyst. In every case product selectivity was $100 \%$ and only in case of $\mathrm{m}$-Xylene, Toluene, Ethyl benzene and anisole two products were obtained.

molar ratio of water and hydrochloric acid in the synthesis gel for the incorporation of the optimum amount of iron into the silicate network. The successful incorporation of iron into 2D-hexagonal silicate framework has made the material to be a superior catalyst for Friedel-Craft benzylation and benzoylation reactions. Large surface area, uniform system of mesopores, fine dispersion of Fe-sites at the mesopore surface and high catalytic activity of the 2D-hexagnoal mesoporous iron silicate may confer its paramount importance in other acid catalyzed reactions.

$$
\begin{aligned}
& \text { ABBREVIATIONS } \\
& \text { BET }=\text { Brunauer- Emmett- Teller } \\
& \text { BJH }=\text { Barrett- Joyner-Halenda } \\
& \text { FFT }=\text { Fast Fourier Transform } \\
& \text { FID }=\text { Flame Ionisation Detector } \\
& \text { HMFeS }=\text { Large Mesoporous 2D-Hexagonal Iron Silicate } \\
& \text { PXRD }=\text { Powder X-ray Diffractions } \\
& \text { SDA }=\text { Structure Directing Agent }
\end{aligned}
$$

$$
\begin{array}{ll}
\text { TEOS } & =\text { Tetraethyl orthosilicate } \\
\text { TON } & =\text { Turn Over Number } \\
\text { TVCH } & =1,2,4 \text {-trivinylcyclohexane }
\end{array}
$$

\section{CONFLICT OF INTEREST}

The authors confirm that this article content has no conflicts of interest.

\section{ACKNOWLEDGEMENTS}

$\mathrm{AB}$ wishes to thank the Department of Science and Technology (DST), New Delhi for financial support. SKD and MKB wish to thank to the Council of Scientific and Industrial Research (CSIR), New Delhi for their respective Senior Research Fellowships.

\section{REFERENCES}

[1] Kresge, C. T.; Leonowicz, M. E.; Roth, W. J.; Vartuli, J. C.; Beck, J. S. Ordered mesoporous molecular-sieves synthesized by liquidcrystal template mechanism. Nature, 1992, 359, 710-712.

[2] Beck, J. S.; Vartuli, J. C.; Roth, W. J.; Leonowicz, M. E.; Kresge, C.T.; Schmitt, K. D.; Chu, C. T. W.; Olson, D. H.; Sheppard, E. W.; McCullen, S. B.; Higgins, J. B.; Schlenke, J. L. A new family 
of mesoporous molecular-sieves prepared with liquid crystal templates. J. Am. Chem. Soc., 1992, 114, 10834-1084.

[3] Yoshitake, H.; Sugihara, T.; Tatsumi, T. Preparation of wormholelike mesoporous $\mathrm{TiO} 2$ with an extremely large surface area and stabilization of its surface by chemical vapor deposition. Chem. Mater., 2002, 14, 1023-1029.

[4] Banerjee, S.; Santhanam, A.; Dhathathreyan, A.; Rao, P. M. Synthesis of ordered hexagonal mesostructured nickel oxide. Langmuir, 2003, 19, 5522-5525.

[5] Park, H.; Yang, S. H.; Jun, Y. -S.; Hong, W. H.; Kang, J. K. Facile route to synthesize large-mesoporous gamma-alumina by room temperature ionic liquids. Chem. Mater., 2007, 19, 535-542.

[6] Sinha, A. K.; Suzuki, K. Three-Dimensional mesoporous chromium oxide: a highly efficient material for the elimination of volatile organic compounds. Angew. Chem. Int. Ed. Engl., 2005, 44, 271-273.

[7] Das, S. K.; Bhunia, M. K.; Sinha, A. K.; Bhaumik, A. Synthesis, characterization and biofuel application of mesoporous Zirconium Oxophosphates. ACS Catal., 2011, 1, 493-501.

[8] Sayle, D. C.; Mangili, B. C.; Klinowski, J.; Sayle, T. X. T. Simulating self-assembly of $\mathrm{ZnS}$ nanoparticles into mesoporous materials. J. Am. Chem. Soc., 2006, 128, 15283-15291.

[9] Han, Z.; Zhu, H.; Shi, J.; Parkinson, G.; Lu, G. Q. Preparation of mesoporous cadmium sulfide nanoparticles with moderate pore size. J. Solid State Chem., 2007, 180, 902-906.

[10] Tiemann, M.; Fröba, M. Mesostructured aluminophosphates synthesized with supramolecular structure directors. Chem. Mater., 2001, 13, 3211-3217.

[11] Mal, N. K.; Bhaumik, A.; Fujiwara, M.; Matsukata, M. Syntheses of mesoporous hybrid iron oxophenyl phosphate, iron oxophosphate, and sulfonated oxophenyl phosphate. Ind. Eng. Chem. Res., 2006, 45, 7748-7751.

[12] Tarafdar, A.; Biswas, S.; Pramanik, N. K.; Pramanik, P. Synthesis of mesoporous chromium phosphate through an unconventional sol-gel route. Microporous Mesoporous Mater., 2006, 89, 204-208.

[13] Sarkar, K.; Nandi, M.; Bhaumik, A. Enhancement in microporosity and catalytic activity on grafting silica and organosilica moieties in lamellar titanium phosphate framework. Appl. Catal. A. Gen., 2008, 343, 55-61.

[14] Das, S. K.; Nandi, M.; Giri, S.; Bhaumik, A. A new mesoporous $\mathrm{FeBO}_{3}$ material having dominant surface magnetism. Microporous Mesoporous Mater., 2009, 117, 362-367.

[15] Boyapati, M.; Choudary, B. M.; Mulukutla, R. S.; Klabunde, K. J. Benzylation of aromatic compounds with different crystallites of MgO. J. Am. Chem. Soc., 2003, 125, 2020-2021.

[16] Ayyappan, S.; Rao, C. N. R. Mesoporous aluminoborates. Chem. Commun., 1997, (6), 575-576.

[17] Iwanami, K.; Choi, J.-C.; Lu, B.; Sakakura, T.; Yasuda, H. Remarkable acceleration of cyanosilylation by the mesoporous AlMCM-41 catalyst. Chem. Commun. (Camb.), 2008, (8), 1002-1004.

[18] Li, Y.; An, D.; Zhang, Q.; Wang, Y. Copper-Catalyzed selective oxidation of Methane by Oxygen: studies on catalytic behavior and functioning mechanism of $\mathrm{CuO}_{x} / \mathrm{SBA}-15$. J. Phys. Chem. C., 2008, 112, 13700-13708.

[19] Yu, C.; Dong, X.; Guo, L.; Li, J.; Qin, F.; Zhang, L.; Shi, J.; Yan, D. Template-Free preparation of mesoporous $\mathrm{Fe}_{2} \mathrm{O}_{3}$ and its application as absorbents. J. Phys. Chem. C., 2008, 112, 1337813382

[20] Zhao, D.; Feng, J.; Huo, Q.; Melosh, N.; Fredrickson, G. H.; Chmelka, B. F.; Stucky, G. D. Triblock copolymer syntheses of mesoporous silica with periodic 50 to 300 angstrom pores. Science, 1998, 279, 548-552.

[21] Samanta, S.; Giri, S.; Sastry, P. U.; Mal, N. K.; Manna, A.; Bhaumik, A. Synthesis and characterization of iron-rich highly ordered mesoporous Fe-MCM-41. Ind. Eng. Chem. Res., 2003, 42, 3012-3018.

[22] Dufaud, V.; Davis, M. E. Design of heterogeneous catalysts via multiple active site positioning in organic-inorganic hybrid materials. J. Am. Chem. Soc., 2003, 125, 9403-9413.

[23] Liu, Y. M.; Cao, Y.; Zhu, K. K.; Yan, S. R.; Dai, W. L.; He, H. Y.; Fan, K. N. Highly efficient $\mathrm{VO}_{x} / \mathrm{SBA}-15$ mesoporous catalysts for oxidative dehydrogenation of propane. Chem. Commun., 2002, (23), 2832-2833.

[24] Liu, Y. M.; Cao, Y.; Yi, N.; Feng, W. L.; Yan, S. R.; Dai, W. L.; He, H. Y.; Fan, K. N. Vanadium oxide supported on mesoporous
SBA-15 as highly selective catalysts in the oxidative dehydrogenation of propane. J. Catal., 2004, 224, 417-428.

[25] Taguchi, A.; Schüth, F. Ordered mesoporous materials in catalysis. Microporous Mesoporous Mater., 2005, 77, 1-45.

[26] Groothaert, M. H.; van Bokhoven, J. A.; Battiston, A. A.; Weckhuysen, B. M.; Schoonheydt, R.A. Bis(mu-oxo)dicopper in $\mathrm{Cu}-\mathrm{ZSM}-5$ and its role in the decomposition of NO: a combined in situ XAFS, UV-Vis-Near-IR, and kinetic study. J. Am. Chem. Soc., 2003, 125, 7629-7640.

[27] Groothaert, M. H.; Smeets, P. J.; Sels, B. F.; Jacobs, P. A.; Schoonheydt, R. A. Selective oxidation of methane by the bis(muoxo)dicopper core stabilized on ZSM-5 and mordenite zeolites. $J$. Am. Chem. Soc., 2005, 127, 1394-1395.

[28] Jarry, B.; Launay, F.; Nogier, J. P.; Montouillout, V.; Gengembre, L.; Bonardet, J. L. Characterisation, acidity and catalytic activity of Ga-SBA-15 materials prepared following different synthesis procedures. Appl. Catal. A. Gen., 2006, 309, 177-186.

[29] Yue, Y.; Gideon, A.; Bonardet, J. L.; Melosh, N.; DøEspinose, J. B.; Fraissard, J. Direct synthesis of A1SBA mesoporous molecular sieves: characterization and catalytic activities. Chem. Commun., 1999, (19), 1967-1968.

[30] Vinu, A.; Sawant, D. P.; Ariga, K.; Hossain, K. Z.; Halligudi, S. B.; Hartmann, M.; Nomura, M. Direct synthesis of well-ordered and unusually reactive FeSBA-15 mesoporous molecular sieves. Chem. Mater., 2005, 17, 5339-5345.

[31] Nozaki, C.; Lugmair, C. G.; Bell, A. T.; Tilley, T. D. Synthesis, characterization, and catalytic performance of single-site iron(III) centers on the surface of SBA-15 silica. J. Am. Chem. Soc., 2002, 124, 13194-13203.

[32] Bhaumik, A.; Samanta, S.; Mal, N. K. Iron oxide nanoparticles stabilized inside highly ordered mesoporous silica. PRAMANA J. Phys., 2005, 65, 855-862.

[33] Das, S. K.; Bhunia, M. K.; Bhaumik, A. Highly ordered Ti-SBA15: Efficient $\mathrm{H}_{2}$ adsorbent and photocatalyst for eco-toxic dye degradation. J. Solid State Chem., 2010 183, 1326-1333.

[34] Li, Y.; Feng, Z. C.; Lian, Y. X.; Sun, K. Q.; Zhang, L.; Jia, G. Q.; Yang, Q. H.; Li, C. Direct synthesis of highly ordered Fe-SBA-15 mesoporous materials under weak acidic conditions. Microporous Mesoporous Mater., 2005, 84, 41-49.

[35] Byambajav, E.; Ohtsuka, Y. Hydrocracking of asphaltene with metal catalysts supported on SBA-15. Appl. Catal. A. Gen., 2003, 252, 193-204.

[36] Wang, X.; Zhang, Q.; Guo, Q.; Lou, Y.; Yang, L.; Wang, Y. Ironcatalysed propylene epoxidation by nitrous oxide: dramatic shift of allylic oxidation to epoxidation by the modification with alkali metal salts. Chem. Commun. (Camb.), 2004, (12), 1396-1397.

[37] Murugavel, R.; Roesky, H. W. Titanosilicates: recent developments in synthesis and use as oxidation catalysts. Angew. Chem. Int. Ed. Engl., 1997, 36, 477-479.

[38] Olah, G. A. Friedel-Crafts Chemistry, Wiley: New York, 1973.

[39] Commandeur, R.; Berger, N.; Jay, P.; Kervennal, J. (Benzyl)methyl-substd. Phenylene-methylene oligomer mixt.|comprises oligomer of benzyl toluene and oligomer of benzyl:xylene. European Patent 0442986, 1991.

[40] Barlow, S. J.; Clark, J. H.; Darby, M. R. Friedel-Crafts alkylation using montmorillonite-supported $\mathrm{ZnCl}_{2}, \mathrm{CuCl}_{2}$, and $\mathrm{MgCl}_{2}$ catalysts. J. Chem. Res., 1991, 3, 74-75.

[41] Khadilkar, B. M.; Borkar, S. D. Environmentally clean synthesis of diphenylmethanes using silica gel-supported $\mathrm{ZnCl}_{2}$ and $\mathrm{FeCl}_{3}$. J. Chem. Technol. Biotechnol., 1998, 71, 209-212.

[42] Choudhary, V. R.; Jana, S. K.; Kiran, B. P. Highly active and moisture-insensitive solid catalysts $-\mathrm{GaCl}_{3}$ and $\mathrm{InCl}_{3}$ supported on montmorillonite-K10 and Si-MCM-41 for benzylation of benzene. Catal. Lett., 2000, 64, 223.

[43] Choudhary, V. R.; Jana, S. K. Benzoylation of benzene and substituted benzenes by benzoyl chloride over $\operatorname{In}_{2} \mathrm{O}_{3} / \mathrm{Si}-\mathrm{MCM}-41$ catalyst. J. Mol. Catal. A. Chem., 2002, 180, 267-276.

[44] Choudhary, V. R.; Jana, S. K.; Mamman, A. S. Benzylation of benzene by benzyl chloride over Fe-modified ZSM-5 and H-beta zeolites and $\mathrm{Fe}_{2} \mathrm{O}_{3}$ or $\mathrm{FeCl}_{3}$ deposited on micro-, meso- and macroporous supports Microporous Mesoporous Mater., 2002, 56, 65-71.

[45] Hu, X.; Chuah, G. K.; Jaenicke, S. Room temperature synthesis of diphenylmethane over MCM-41 supported $\mathrm{AlCl}_{3}$ and other Lewis acids. Appl. Catal. A. Gen., 2001, 217, 1-9. 
[46] Chiu, J. J.; Pine, D. J.; Bishop, S. T.; Chmelka, B. F. Friedel-Crafts alkylation properties of aluminosilica SBA-15 meso/macroporous monoliths and mesoporous powders. J. Catal., 2004, 221, 400-412.

[47] Chandra, D.; Das, S. K.; Bhaumik. A. A fluorophore grafted 2Dhexagonal mesoporous organosilica: excellent ion-exchanger for the removal of heavy metal ions from wastewater. Microporous Mesoporous Mater., 2010, 128, 3-40.

[48] Wu, C.; Kong, Y.; Gao, F.; Wu, Y.; Lu, Y.; Wang, J.; Dong, L. Synthesis, characterization and catalytic performance for phenol hydroxylation of Fe-MCM41 with high iron content. Microporous Mesoporous Mater., 2008, 113, 163-170.

[49] Solsona, B.; Blasco, T.; Nieto, J. M. L.; Peña, M. L.; Rey, F.; Vidal- Moya, A. Vanadium oxide supported on mesoporous MCM-
41 as selective catalysts in the oxidative dehydrogenation of alkanes. J. Catal., 2001, 203, 443-452.

[50] Jin, C.; Li, G.; Wang, X.; Zhao, L.; Liu, L.; Haiou, H.; Liu, L.; Zhang, W.; Han, X.; Bao, X. Synthesis, characterization and catalytic performance of Ti-containing mesoporous molecular sieves assembled from Titanosilicate precursors. Chem. Mater., 2007, 19, 1664-1670.

[51] Wu, C.; Kong, Y.; Gao, F.; Wu, Y.; Lu, Y.; Wang, J.; Dong, L. Synthesis, characterization and catalytic performance for phenol hydroxylation of Fe-MCM41 with high iron content. Microporous Mesoporous Mater., 2008, 113, 163-170.

[52] Sereda, G. A. Alkylation on graphite in the absence of Lewis acids. Tetrahedron Lett., 2004, 45, 7265-7267.

(C) Das et al.; Licensee Bentham Open.

This is an open access article licensed under the terms of the Creative Commons Attribution Non-Commercial License (http://creativecommons.org/licenses/by-nc/ 3.0/) which permits unrestricted, non-commercial use, distribution and reproduction in any medium, provided the work is properly cited. 